OPEN ACCESS

Edited by:

Jürgen Soll,

Ludwig Maximilian University

of Munich, Germany

Reviewed by:

Zach Adam,

Hebrew University of Jerusalem, Israe

Christian Schmitz-Linneweber,

Humboldt-Universität zu Berlin,

Germany

*Correspondence:

Yafei Q

Yafei.Qi@nwsuaf.edu.cn

tThese authors have contributed equally to this work

Specialty section:

This article was submitted to Plant Physiology,

a section of the journal

Frontiers in Plant Science

Received: 10 December 2018

Accepted: 22 February 2019

Published: 12 March 2019

Citation:

Liu S, Zheng L, Jia J, Guo J, Zheng M, Zhao J, Shao J, Liu X, An L,

Yu F and Qi Y (2019) Chloroplast

Translation Elongation Factor

EF-Tu/SVR11 Is Involved

in var2-Mediated Leaf Variegation and Leaf Development in Arabidopsis.

Front. Plant Sci. 10:295.

doi: $10.3389 /$ fpls.2019.00295

\section{Chloroplast Translation Elongation Factor EF-Tu/SVR11 Is Involved in var2-Mediated Leaf Variegation and Leaf Development in Arabidopsis}

\author{
Siyu Liu't, Lu Zhengt, Jia Jia, Jia Guo, Mengdi Zheng, Jun Zhao, Jingxia Shao, \\ Xiayan Liu, Lijun An, Fei Yu and Yafei Qi* \\ State Key Laboratory of Crop Stress Biology for Arid Areas and College of Life Sciences, Northwest A\&F University, \\ Yangling, China
}

Chloroplasts are semiautonomous organelles, retaining their own genomes and gene expression apparatuses but controlled by nucleus genome encoded protein factors during evolution. To analyze the genetic regulatory network of $\mathrm{FtsH}$-mediated chloroplast development in Arabidopsis, a set of suppressor mutants of yellow variegated (var2) have been identified. In this research, we reported the identification of another new var2 suppressor locus, SUPPRESSOR OF VARIEGATION11 (SVR11), which encodes a putative chloroplast-localized prokaryotic type translation elongation factor EF-Tu. SVR11 is likely essential to chloroplast development and plant survival. GUS activity reveals that SVR11 is abundant in the juvenile leaf tissue, lateral roots, and root tips. Interestingly, we found that SVR11 and SVR9 together regulate leaf development, including leaf margin development and cotyledon venation patterns. These findings reinforce the notion that chloroplast translation state triggers retrograde signals regulate not only chloroplast development but also leaf development.

Keywords: EF-Tu, chloroplast development, leaf variegation, retrograde signal, VAR2

\section{INTRODUCTION}

Chloroplasts are essential organelles for eukaryotic photosynthetic species, enabling the chemical reactions powered by light energy to reduce $\mathrm{CO}_{2}$ to carbohydrates. It is believed that chloroplasts evolved from ancient prokaryotic cyanobacteria through endosymbiosis (Martin et al., 2002). This co-evolution process, especially the transfer of most genes of chloroplast progenitors to the host nuclear genomes, have given rise to modern-day chloroplast genomes with only around 120 genes, in contrast to the more than 3,000 genes of the current genome of cyanobacteria, such as Synechocystis sp. (Timmis et al., 2004). The physical separation of nuclear and chloroplast genomes raises at least two important implications. First, the remaining genes in chloroplast genomes are expressed with prokaryotic gene expression systems, which are regulated by the nuclear genome and must respond to developmental and environmental conditions (Jarvis and López-Juez, 2013).

Abbreviations: CaMV, Cauliflower Mosaic Virus; EF-Ts, elongation factor thermo stable; EF-Tu, elongation factor thermo unstable; FtsH, filamentous temperature sensitive H; GFP, green fluorescence protein; SVR, SUPPRESSOR OF VARIEGATION; UTR, untranslated region; var2, yellow variegated2. 
Second, many photosynthetic protein complexes are chimeric in nature, and are composed of subunits encoded by both nuclear and plastid genomes, and intricate regulation at different levels are necessary for the optimal assembly of these complexes. The semi-autonomous nature of the chloroplast thus necessitates a fine coordination between the two genomes (Kleine and Leister, 2016).

Higher plants have evolved multiple strategies to facilitate the expression and coupling of nuclear and chloroplast genomes. At the translation level, chloroplasts utilize a prokaryotic translation system featuring the $70 \mathrm{~S}$ ribosome (Yamaguchi and Subramanian, 2000; Yamaguchi et al., 2000; Tiller and Bock, 2014). Prokaryotic translation initiates through the binding of $30 \mathrm{~S}$ ribosomal subunit to the Shine-Dalgarno sequence of mRNA, and the subsequent association of initiator tRNA leads to the formation of pre-initiation complex, and this process is assisted by initiation factors IFs (Laursen et al., 2005). In Arabidopsis, chloroplast IF1 and IF2 homologs are encoded by the nuclear genome, and null mutant alleles of Arabidopsis IF1 and IF2 are embryonic lethal, indicating they are essential genes for plant viability (Miura et al., 2007; Shen et al., 2013; Nesbit et al., 2015). Further recruitment of the 50S ribosomal subunit to pre-initiation complex forms an active initiation complex (Laursen et al., 2005). The translation process requires elongation factors EF-Tu, EF-G, and EF-Ts to incorporate aminoacyl-tRNAs into $70 \mathrm{~S}$ ribosomes (Krab and Parmeggiani, 2002). EF-Tu is a prokaryotic elongation factor belonging to the GTP-binding protein family (Krab and Parmeggiani, 2002). During translation elongation, GTP-bound EF-Tu forms a ternary complex with aminoacyl-tRNA to facilitate the transport of cognate aminoacyl-tRNA to the A-site of the 70S ribosome. Next, the innate GTPase activity of EF-Tu hydrolyzes the GTP to GDP, and GDP-bound EF-Tu is released from ribosome and recycled to GTP-bound EF-Tu mediated by EF-Ts for the next round of elongation (Krab and Parmeggiani, 1998). During endosymbiosis, genes coding for many of the chloroplast $70 \mathrm{~S}$ ribosomal proteins and most translational factors have been transferred to the nuclear genome and are subject to nuclear regulation. Partial loss of chloroplast EF-Tu activities in Arabidopsis, maize, and tomato reduced heat tolerance, suggesting chloroplast EF-Tu is involved in the plant response to environmental changes (Ristic et al., 2004; Li et al., 2018).

Chloroplast gene expression is also regulated by posttranslational mechanisms including the operation of a vast array of protease systems (Nishimura et al., 2016). An intriguing group of proteolytic enzymes that has attracted attention is the chloroplast FtsH proteases, due to the unique leaf variegation phenotypes of yellow variegated1 (var1) and yellow variegated2 (var2) mutants, defective in thylakoid-localized Fts $\mathrm{H}$ proteins AtFtsH5 and AtFtsH2, respectively (Chen et al., 2000; Takechi et al., 2000; Sakamoto et al., 2002). FtsH proteins belong to the AAA (ATPase associated with various cellular activities) ATPase superfamily, which is ubiquitously present in prokaryotes and eukaryotes, as well as in mitochondria and chloroplasts (Janska et al., 2013). Thylakoid FtsH complexes comprise four members of FtsH proteins, FtsH1 and FtsH5 (type A) and FtsH2 and FtsH8 (type B), in which VAR2/AtFtsH2 is one of the most abundant subunits (Yu et al., 2004; Zaltsman et al., 2005; Nishimura et al., 2016). Biochemical analysis suggested that thylakoid FtsHs are required to degrade photodamaged reaction center protein D1 during Photosystem II repair cycle (Lindahl et al., 2000; Kato et al., 2009; Malnoë et al., 2014). Interestingly, VAR2/AtFtsH2-mediated post-translational regulation is closely related with chloroplast translation. Multiple genetic screens for var2 suppressors in several laboratories have yielded an increasing number of genetic factors involved in chloroplast transcription, translation and post-translational turnover (Park and Rodermel, 2004; Yu et al., 2008; Adam et al., 2011; reviewed in Liu et al., 2010 and Putarjunan et al., 2013). Recently, we reported a new var2 suppressor mutant, svr91 , which is defective in a bona fide chloroplast-localized prokaryotic translation initiation factor IF3 (Zheng et al., 2016). In Bacteria, initiation factor IF3, encoded by the essential infC gene, binds to the $30 \mathrm{~S}$ ribosomal subunit to promote dissociation of the $70 \mathrm{~S}$ ribosome for ribosome recycling and translation initiation (Laursen et al., 2005). Down regulation of SVR9, alone or with its homologous gene SVR9L1, not only suppresses var2 leaf variegation phenotype, but also causes leaf developmental abnormalities including serrated leaf margin and altered cotyledon venation patterns (Zheng et al., 2016). The characterization of var2 suppressor genes thus provides a unique opportunity to uncover additional regulators of chloroplast translation.

Here, we report the identification of a new var2 suppressor mutant, svr11-1. Molecular cloning, complementation and protein localization studies confirmed that SVR11 encodes a putative prokaryotic translation elongation factor EF-Tu, which is localized in chloroplasts. Interestingly, functional genetic analysis of SVR11, SVR9, and SVR9L1 showed that svr11-1 svr9-1 double mutants display a more serrated leaf margin and altered cotyledon venation patterns compared to those of the wild type, while svr11-1 svr9-1 svr9-1l-1/+ mutants have an even more pronounced leaf serration. These data suggest that chloroplast translation elongation factor EF-Tu/SVR11 not only regulate chloroplast development, but also act synergistically with chloroplast translation initiation factor IF3/SVR9 to dictate leaf margin and cotyledon vascular development. Our findings uncover a new translation elongation factor in regulating chloroplast and leaf development in Arabidopsis.

\section{MATERIALS AND METHODS}

\section{Plant Materials and Growth Conditions}

Arabidopsis thaliana plants used in this study are all in the Columbia-0 background. The T-DNA insertion line CS819179 (svr11-3) was obtained from the Arabidopsis Biological Resource Center (ABRC); the accurate position of each T-DNA insertion sites were identified by sequencing PCR products that include plant genomic DNA and T-DNA left border sequences. Arabidopsis seeds were grown at $22^{\circ} \mathrm{C}$ under continuous illumination $\left(\sim 100 \mu \mathrm{mol} \mathrm{m} \mathrm{m}^{-2} \mathrm{~s}^{-1}\right)$ on commercial soil mix (Pindstrup, Denmark). All seeds were stratified for 2 days at $4^{\circ} \mathrm{C}$ before sown on soil or half strength $\mathrm{MS}$ medium. For 
heat stress at a moderate level, 8-day-old seedlings were treated at $38^{\circ} \mathrm{C}$ for $90 \mathrm{~min}$, and then moved into $22^{\circ} \mathrm{C}$ for recovery (Queitsch et al., 2000).

\section{Chlorophyll Fluorescence Imaging}

Chlorophyll fluorescence was measured with 2-week-old plants using Open FluorCam FC800-O (Photon Systems Instruments; Czechia). Whole plants were dark adapted for $10 \mathrm{~min}$ to oxidize the plastoquinone pool before measurement, and the minimum fluorescence $F_{\mathrm{O}}$ was measured. The maximum fluorescence $F_{\mathrm{M}}$ was determined by a saturating flash of light. The maximum quantum yield of photosystem II $\left(F_{\mathrm{V}} / F_{\mathrm{M}}\right)$ is calculated as $F_{\mathrm{M}}-F_{\mathrm{O}} / F_{\mathrm{M}}$. Measurement of $F_{\mathrm{V}} / F_{\mathrm{M}}$ was performed in three independent biological repeats.

\section{RNA Manipulations, Vector Constructions, and Transformations}

Total RNAs were extracted using Trizol RNA reagent (Life Technologies, Carlsbad, CA, United States) according to the manufacturer's instructions. For semi-quantitative RT-PCR analysis, first-strand cDNA was synthesized from $1 \mu \mathrm{g}$ DNasetreated total RNA using a PrimeScript reverse transcription kit (Roche, Switzerland). The gene-specific primers used in this study are listed in Table 1. The semi-quantitative RT-PCR was performed in three independent biological repeats.

To complement the svr11-1 and 049-002 mutants, a full-length At4g20360 (SVR11) cDNA was amplified by Primer STAR ${ }^{\text {TM }}$ HS DNA polymerase (Takara) using primers 20360F and 20360R. The PCR product was digested with BamHI and cloned into the BamHI site of pBluescript KS+. The sequenced SVR11 fragment was then transferred into pBI111L-intron plasmid which is modified from pBI111L plasmid (Yu et al., 2004). In brief, the first intron sequence of At5g27700 at its 5' UTR region were amplified with primers 27700inF (XbaI) and 27700inR
$(B a m \mathrm{HI})$, and inserted into the multiple cloning site of $\mathrm{pBI} 111 \mathrm{~L}$ as a chimeric intron at the $5^{\prime}$ UTR region of insertion genes. The resulting construct was transformed into Agrobacterium by electroporation. Arabidopsis transformation was performed as described (Clough and Bent, 1998).

\section{Inverse PCR}

Genomic DNA extracted from 049-002 homozygous plants was digested with restriction enzyme EcoRI overnight. The DNA fragments were further precipitated with 2.5 volumes of ethanol and 0.1 volumes of $3 \mathrm{M}$ sodium acetate ( $\mathrm{pH}$ 5.2). After dissolving in Milli-Q water, DNA fragments were ligated with T4 DNA Ligase. Inverse PCR were performed with Pfu DNA Polymerase using primers SKC12 and OCS3. One $1.5 \mathrm{~kb}$ PCR amplified band was sequenced with the SKC12 primer.

\section{Evolutionary Analysis}

Full-length protein sequences of SVR11 homologous proteins from dicots Arabidopsis thaliana, monocots Oryza sativa, moss Physcomitrella patens, green algae Chlamydomonas reinhardtii, yeast Saccharomyces cerevisiae, and prokaryotic species such as Synechocystis and Escherichia coli were obtained from NCBI using the BLASTP program. Evolutionary analyses were conducted in MEGA X, and the Neighbor-joining algorithm was used to generate the initial tree (Kumar et al., 2018). The accession numbers of protein sequences were included.

\section{Transient Expression of SVR11-GFP and SVR11-Like-GFP}

In order to generate a C-terminal GFP-tagged SVR11, the coding sequences of SVR11 amplified with primers 20360F and 20360 GFPR, SVR11-like with 02930F and 02930 GFPR, were cloned into transient expression vector pTF486. The resulting construct were designated p35S::SVR11-GFP and p35S::SVR11-like-GFP.

\begin{tabular}{|c|c|c|}
\hline $20360 \mathrm{~F}$ & 5'-CATGGATCCACCCTAGCTTCTCGATTTCTC-3' & p35S:: intronSVR11 \\
\hline $27700 \mathrm{inF}$ & 5'-CATCTCGAGACTCTCGCTTTCTTCATCATCTC-3' & p35S:: intronSVR11 \\
\hline $27700 \mathrm{inR}$ & 5'-CATTCTAGAGCTTTGAAAGAGTAAACGAGTCC-3' & \\
\hline 02930 GFPR & 5'-CGCGGATCCGGTCATCACTITGATACAAC-3' & \\
\hline $20360 \mathrm{PF}$ & 5'-CATTCTAGACTACCCTITGCTGTCTTGTAAG-3' & pSVR11::uidA \\
\hline 20360 PR & 5'-CATGGATCCGAAGATGGAATTGGAGAGCAGAG-3' & \\
\hline 20360 F3 & 5'-GTTACGATTTGTGACGTGTG-3' & Genotyping \\
\hline $20360 \mathrm{~F} 1$ & 5'-ACСCTAGCTTCTCGATTTCTC-3' & \\
\hline uidA-R & 5'-GTTCAGTTCGTTGTTCACAC-3' & Genotyping \\
\hline SKC12 & $5^{\prime}$-TTGACAGTGACGACAAATCG-3' & Inverse PCR \\
\hline ocs3 & 5'-TAGAGCTCTTATACTCGAGG-3' & Inverse PCR \\
\hline
\end{tabular}


Arabidopsis leaf protoplast preparation and transient expression of GFP constructs were performed as described by Yoo et al. (2007). Bright field images and fluorescent signals from GFP and chlorophyll autofluorescence were monitored using a Leica DM5000B fluorescent microscope (Leica, Germany).

\section{Histochemical GUS Staining}

Amplified with primers 20360 PF and 20360 PR, a 1,493bp genomic DNA fragment upstream of the start codon of At4g20360 was cloned into pCB308 (Xiang et al., 1999), to generate SVR11 promoter- $\beta$-glucuronidase (GUS) construct pSVR11::uidA. The construct pSVR11::uidA was transformed into wild-type Arabidopsis plant, and $p S V R 11:: u i d A$ lines were screened with BASTA. GUS activities were assayed in the T2 generations (Jefferson et al., 1987). The GUS staining was performed with three independent transgenic lines.

\section{Leaf Silhouettes and Cotyledon Veins Observation}

For leaf silhouettes imaging, individual true leaves were covered with water and photographed using Research stereo microscope (SMZ25; Nikon) equipped with a CCD camera (DS-U3; Nikon). Those photos were converted to black and white by Adobe Photoshop 8.0.1 by filled the leaf blades with black, so that it is easier to observe the silhouettes of leaves between different phenotypes. The leaf dissection index was calculated as described (perimeter ${ }^{2} / 4 \pi^{*}$ leaf area, Bilsborough et al., 2011). For cotyledon veins observation, cotyledons of 10-day-old plants were cut down and decolorized in $70 \%$ ethanol, till the cotyledons blade turned colorless without chlorophyll and the veins become clearly visible then the cotyledon samples were photographed by stereo microscope photographing. Cotyledon vein patterns were quantified in three independent biological replicates with tested lines containing at least 100 seedlings each time.

\section{RESULTS}

\section{Identification of 049-002 and svr11-1}

We have performed extensive genetic suppressor screens for mutants that could reverse the var2 leaf variegation phenotype (reviewed in Liu et al., 2010 and Putarjunan et al., 2013). Here, we report the identification of a new recessive suppressor line, designated as 049-002, from the activation tagging T-DNA mutant population in the var2-5 mutant background (Yu et al., 2008). Following our naming sequence, the suppressor gene locus was named as SUPPRESSOR OF VARIEGATION11 (SVR11) and the mutant allele in 049-002 as svr11-1. Overall, 049-002 (var2-5 svr11-1) did not show the characteristic leaf variegation phenotype of var2-5, indicating that svr11-1 is a robust suppressor of var2-5 (Figure 1A). In addition, the statures of 049-002 and svr11-1 resembled that of wild type, suggesting overall plant growth was not dramatically altered by the svr11-1 mutation. Interestingly, both 049-002 and svr11-1 showed a virescent phenotype, i.e., a gradual yellow to green leaf color gradient along the leaf

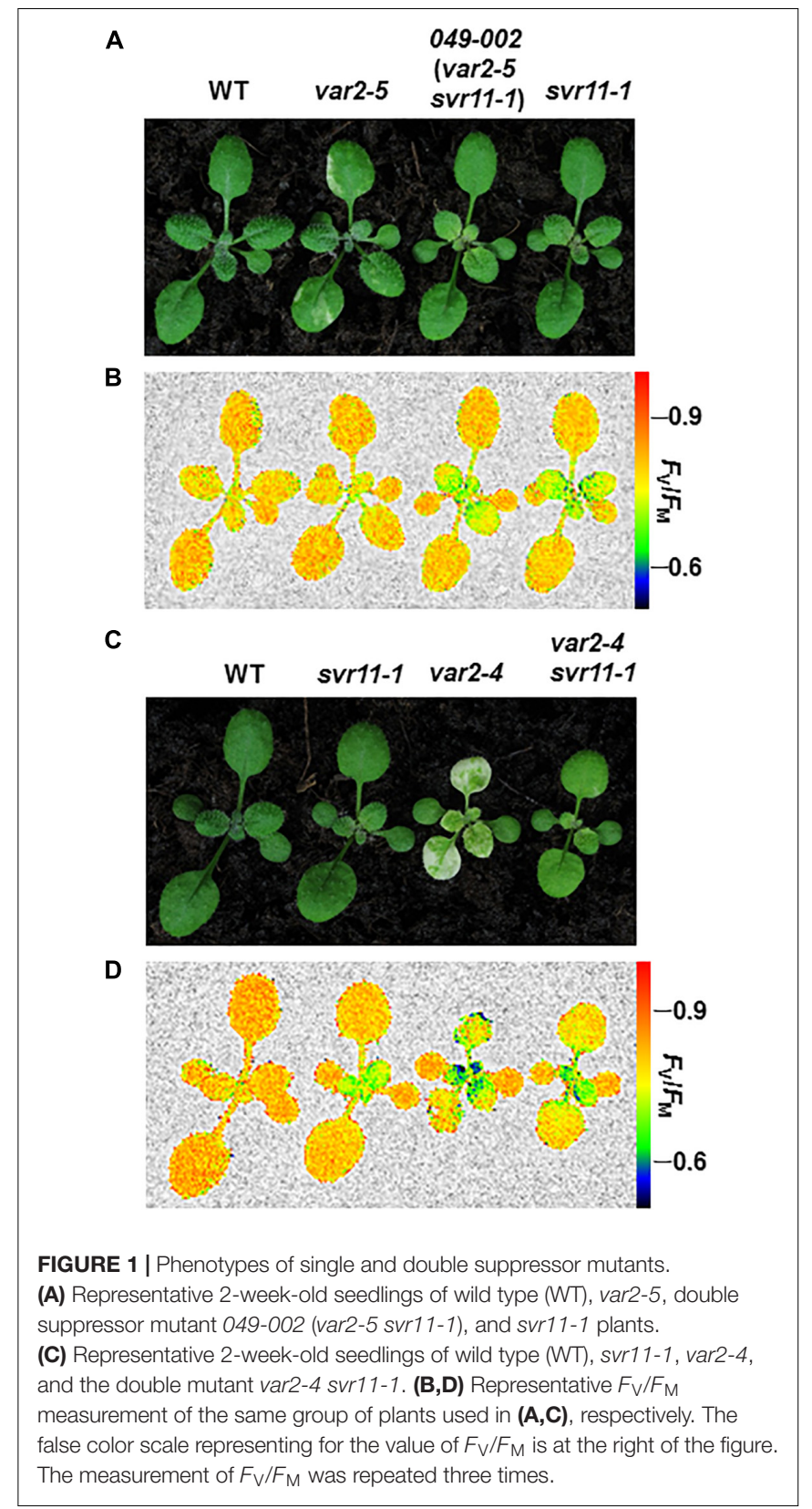

proximal-distal axis (Figure 1A). This virescence phenotype was correlated with a reduction of photosynthetic parameters, as indicated by the $F_{\mathrm{V}} / F_{\mathrm{M}}$ (the maximum quantum yield of photosystem II) of whole plant chlorophyll fluorescence imaging (Figure 1B). svr11-1 could also reverse the leaf variegation of the var2-4 mutant, a stronger allele of var2, indicating that the suppression of var2 leaf variegation by svr11-1 does not depend on the nature of var2 mutation and is not allele specific (Figures 1C,D).

\section{Molecular Cloning of SVR11}

To clone SVR11, we first determined that the svr11-1 mutant phenotype co-segregated with the resistance to herbicide Basta, suggesting the mutant is tagged by T-DNA insert(s) (data not 


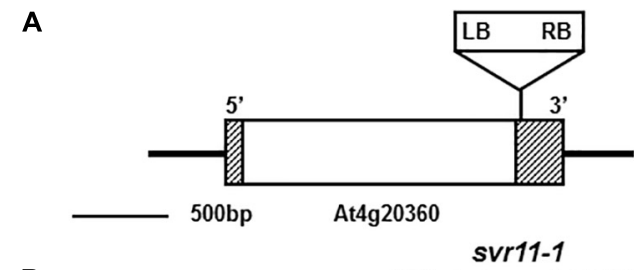

B

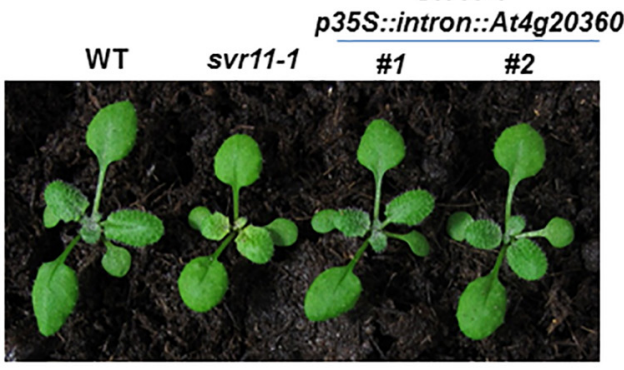

C

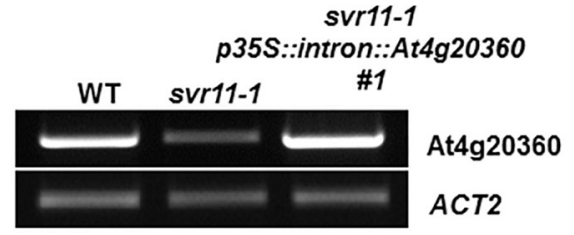

D

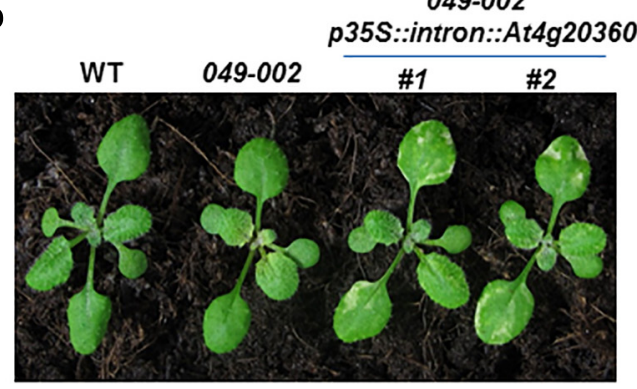

FIGURE 2 | Cloning of SVR11 and complementation of svr11-1. (A) A T-DNA insertion was identified in the $3^{\prime}$-UTR of At4g20360 by inverse PCR. The left border (LB) and the right border (RB) were indicated. Gray boxes and the white box represent UTRs and the exon, respectively. (B) Representative 2-week-old wild-type, svr11-1 and svr11-1 p35S::intron At4g20360 (constitutive expression line of At4g20360 in svr11-1 background). (C) The accumulation of At4g20360 transcripts was indicated by semi-quantitative RT-PCR. The semi-quantitative RT-PCR was repeated three times.

(D) Representative 2-week-old wild-type, 049-002 and 049-002 p35S::intron At4g20360 (constitutive expression of At4g20360 in 049-002 background).

shown). Next, we carried out inverse PCR to identify the T-DNA insertion site and sequencing of inverse PCR products confirmed that the T-DNA was inserted in the $3^{\prime}$ UTR of At4g20360 (Figure 2A). We found that At4g20360 expression was reduced in svr11-1, likely a consequence of T-DNA insertion in $3^{\prime}$ UTR (Figure 2C). Complementation analysis was executed to confirm that the virescent phenotype in svr111 and the suppression of var2 variegation in 049-002 were due to the disruption of SVR11 expression. To this end, we generated a binary vector in which a full-length At4g20360 cDNA was driven by the constitutive CaMV 35S promoter. In addition, sequences of the first intron of At5g27700 were placed between the $35 \mathrm{~S}$ promoter and the cDNA sequences to achieve better expression (Rose et al., 2008). This construct (p35S::intron::At4g20360) was transformed into svr11-1 and 049-002, respectively. We recovered multiple independent transgenic lines and confirmed that elevated expression of At4g20360 was able to complement the virescent phenotype of svr11-1 (Figure 2B). Furthermore, ectopic expression of At4g20360 was able to restore the var2-5 leaf variegation phenotype in 049-002 background (Figure 2D). Together, these data indicate that the virescent chloroplast defect in svr111 and the suppression of var2 leaf variegation in 049-002 were caused by reduced expression of At4g20360, and that At4g20360 is SVR11.

\section{SVR11/At4g20360 Defines a Putative Prokaryotic EF-Tu in Chloroplasts}

Homologous sequences of SVR11 from different species were obtained from National Center for Biotechnology Information (NCBI) and their evolutionary relationship was analyzed (Figure 3A). SVR11 and SVR11-like proteins from these species were all annotated as prokaryotic translation elongation factor EF-Tu homologs. In prokaryotic organisms such as cyanobacteria and E. coli, only one copy of EF-Tu was identified. In contrast, eukaryotic photosynthetic species such as Arabidopsis, rice, moss, and green algae contains at least two EF-Tu homologs (Figure 3A).

The EF-Tu sequences grouped in the same clade with SVR11 in the phylogenetic tree were all predicted to contain chloroplast transit peptides (Figure 3A Clade 2, Emanuelsson et al., 1999). To confirm the sub-cellular localizations of SVR11, a transient expression vector was generated expressing a full-length SVR11 cDNA fused in-frame at its C-terminus with green fluorescent protein (GFP), under the control of the 35S promoter ( $p 35 S:: S V R 11-G F P)$. This construct, as well as a control vector containing only the GFP ( $p 35 S:: G F P)$, were introduced into Wild-type Arabidopsis leaf protoplasts, respectively, and their expressions were monitored by confocal microscopy. Figure 3B shows that GFP signals for p35S::SVR11-GFP appeared as distinct foci, which overlapped nicely with chlorophyll auto-fluorescence signals, suggesting co-localizations with chloroplasts. To examine if SVR11GFP could also be targeted to mitochondria, we transient expressed p35S::SVR11-GFP in protoplasts isolated from transgenic lines stably expressing a mitochondrion marker protein tagged with mCherry, ScCOX4-mCherry (Nelson et al., 2007). SVR11-GFP did not overlap with signals of ScCOX4mCherry, suggesting SVR11-GFP is likely not targeted to mitochondria (Figure 3C). These results demonstrate that the SVR11-GFP is targeted into the chloroplast and SVR11 is a nuclear encoded chloroplast protein. SVR11-like was predicted to be a mitochondrial EF-Tu (Nikolovski et al., 2012), or identified in the mitochondrial soluble protein by mass spectrometry (Ito et al., 2006). Interestingly, SVR11like-GFP aggregate to large or small dots in the cytosol, neither targeted into mitochondria nor to chloroplasts (Supplementary Figure S1). 
A

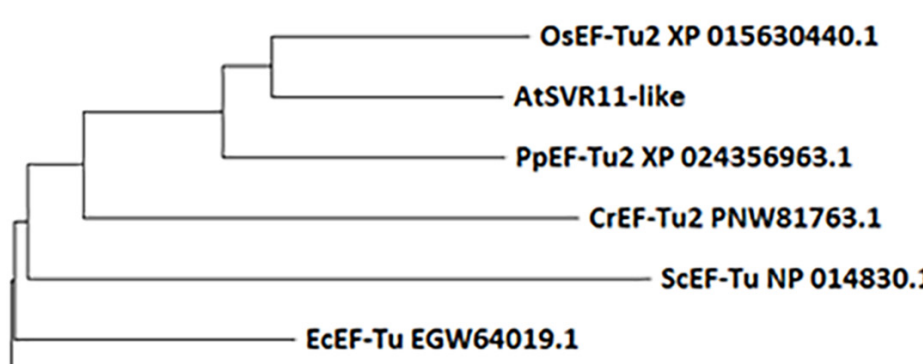

Synechocystis EF-Tu WP 010872941

CrEF-Tu1 NP 958362.1

AtSVR11

OsEF-TU1 XP 015627061.1

PPEF-TU1 XP 024400312.1

\section{Clade 1}

Clade 2
B

GFP
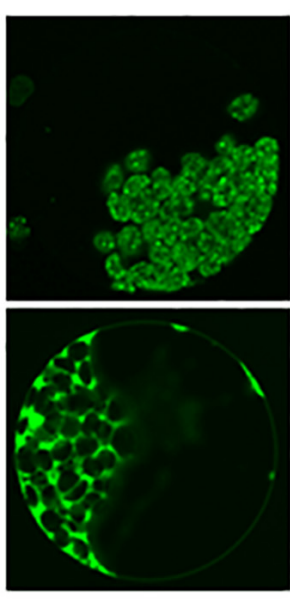

C

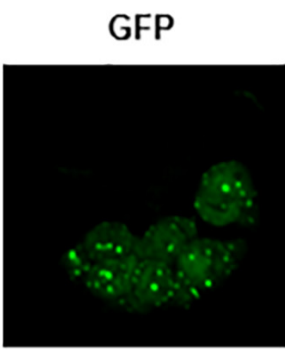

Chlorophyll
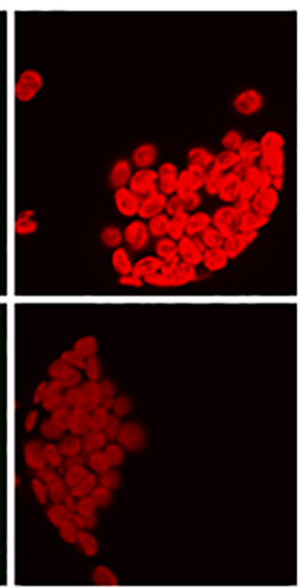

ScCOX4-mCherry

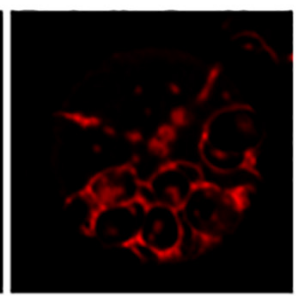

Merge
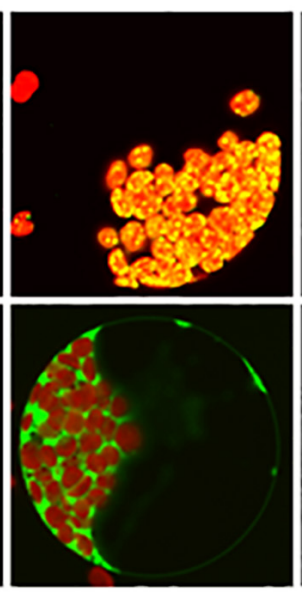

Merge

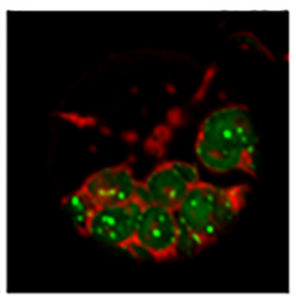

BF

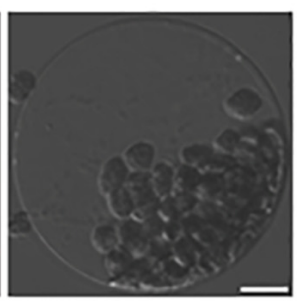

p35S::

SVR11-GFP

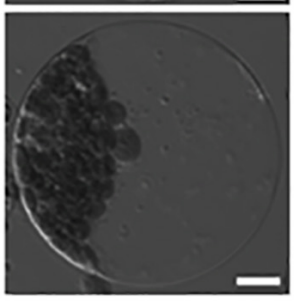

p35S::GFP

BF

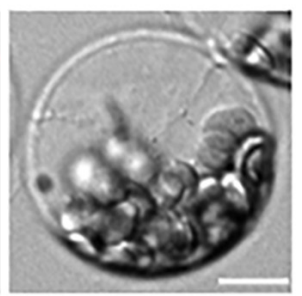

p35S::

SVR11-GFP

FIGURE 3 | Evolutionary relationships of SVR11 homologs and subcellular localization of SVR11. (A) Phylogenetic analysis of the SVR11 protein family. Full-length protein sequences of SVR11 homologous proteins of different species indicated by the accession numbers were obtained from NCBI using BLASTP. The neighbor-joining algorithm was used to generate the initial tree. (B) Leaf protoplasts prepared from wild type Arabidopsis plants were transformed with the p35S::SVR11-GFP fusion construct or the control construct p35S::GFP. Confocal microscopy was used to monitor fluorescence signals from the GFP channel (500-550 nm) and chlorophyll autofluorescence (663-738 nm). Bright field (BF) images served as controls for protoplast integrity. (C) Transient expression of p35S::SVR11-GFP fusion protein in protoplasts isolated from plants expressing a mitochondrion marker ScCOX4-mCherry (570-620 nm). Representative images of a single protoplast are shown. Bar stands for $10 \mu \mathrm{m}$. 


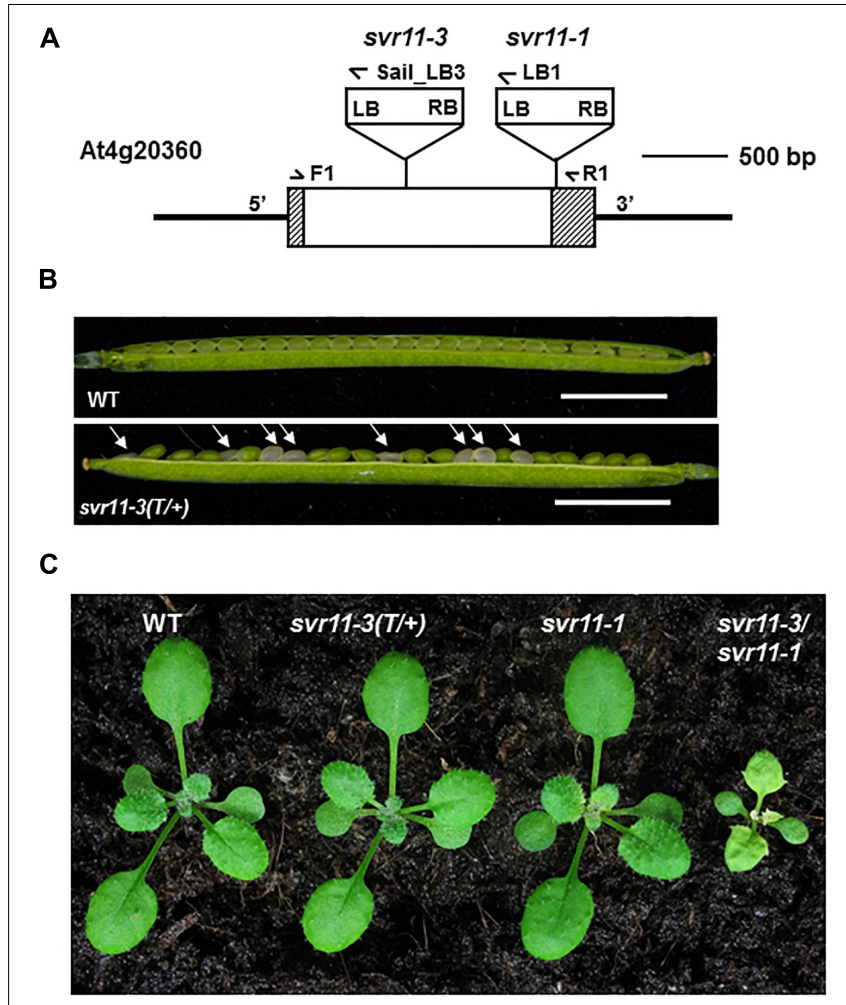

FIGURE 4 | Phenotypic characterization of the svr11 T-DNA insertion null allele mutant. (A) Identification of a null allele of SVR11, svr11-3. (B) Developing seeds within a silique from a heterozygous svr11-3 mutant and wild-type at the same developing state. Bar stands for $2,500 \mu \mathrm{m}$. (C) Representative 2-week-old wild-type, svr11-3(T/+), svr11-1, svr11-3/svr11-1 plants.

\section{Moderate Heat Stress Has Little Impact on var2 Variegations}

It was reported that knock-down of chloroplastic EF-Tu in maize, Arabidopsis, and tomato mutants reduced heat tolerance (Ristic et al., 2004; Li et al., 2018). We then test if heat stress can affect the variegation phenotype of var2 mutants. To avoid lethality caused by the severe heat stress at $45^{\circ} \mathrm{C}$, a moderate level of heat stress at $38^{\circ} \mathrm{C}$ for $90 \mathrm{~min}$ were used as suggested (Queitsch et al., 2000). Moderate heat stress had little impact either on the variegation phenotype of var2 and var2 background suppressor mutants, or on the virescent phenotype of svr11-1 (Supplementary Figure S2).

\section{SVR11 Is Essential to Plant Development}

To further examine the roles that SVR11 play in plant development, we sought for loss-of-function alleles of SVR11. We obtained a second allele of svr11, CS819179 which contained a T-DNA inserted in the encoding sequence of SVR11 (Figure 4A). No homozygous T-DNA insertion line was identified even backcrossed to wild-type five times, probably due to homozygote is embryo lethal. Terminated ovules were observed in the developing siliques in heterozygous mutants (Figure 4B). We renamed CS819179

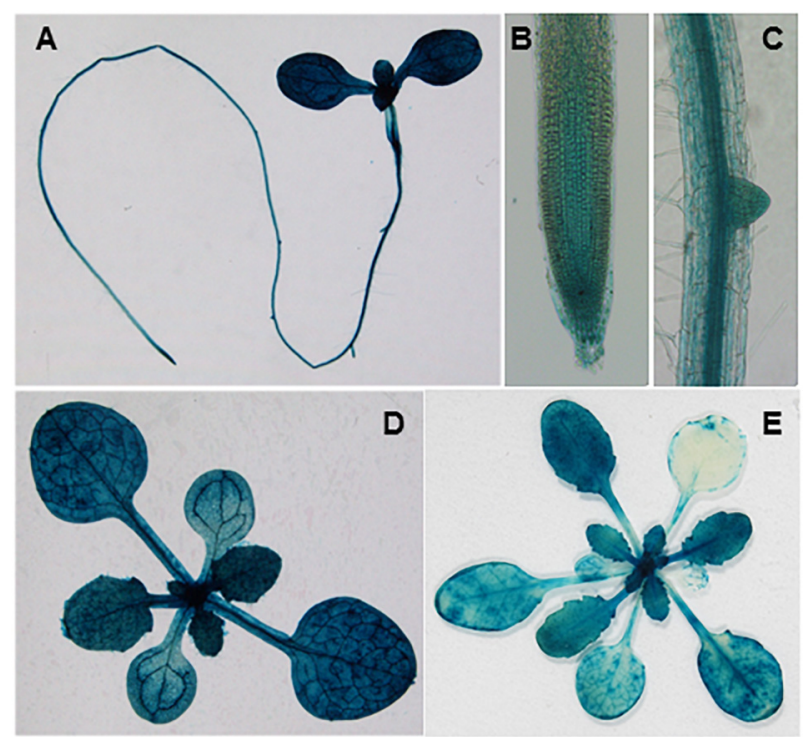

FIGURE 5 | Tissue expression patterns of SVR11. GUS staining of transgenic plants expressing the SVR11 promoter-GUS fusion vector pSVR11::uidA. The GUS staining was performed with three independent transgenic lines. Representative whole seedling (A), root tip (B), and lateral root (C) of 6-day-old transgenic seedling grown on half-strength Murashige and Skoog (MS) solid medium containing 1\% sucrose. Representative leaf tissues above the soil from 2-week-old (D) and 3-week-old (E) plants.

as svr11-3, and then crossed heterozygous svr11-3(T/+) to svr11-1. svr11-1/svr11-3 was obtained by genotyping F1 generation, which is much smaller in size than svr11-1 and the leaf blade is yellow-colored. We speculated that the phenotypic defect severity was determined by SVR11 damage degree (Figure 4C).

\section{SVR11 Is Abundant in Juvenile Tissues}

To characterize the spatial and temporal expression profiles of SVR11, we generated a fusion construct in which the $\beta$-glucuronidase gene (GUS) gene was controlled by the SVR11 promoter (1.5-kb region upstream of the SVR11 start codon). This vector was transformed into wildtype plants and GUS activities of pSVR11:GUS transgenic lines were assayed at different growth stages. In brief, in 6-day-old seedlings GUS expression was detected including in the root tip and lateral roots, suggesting that SVR11 activities are necessary for both photosynthetic and non-photosynthetic tissues (Figures $\mathbf{5 A - C}$ ), also in 2-week-old plants and 3-week-old juvenile rosette leaves (Figures 5D,E).

\section{Chloroplast EF-Tu and IF3 Regulate Leaf Development}

Previously, we have reported a var2 suppressor locus SVR9, encoding a chloroplast translation initiation factor IF3, which mediates var2 leaf variegation and leaf marginal serration formation (Zheng et al., 2016). Next, we tested genetically 


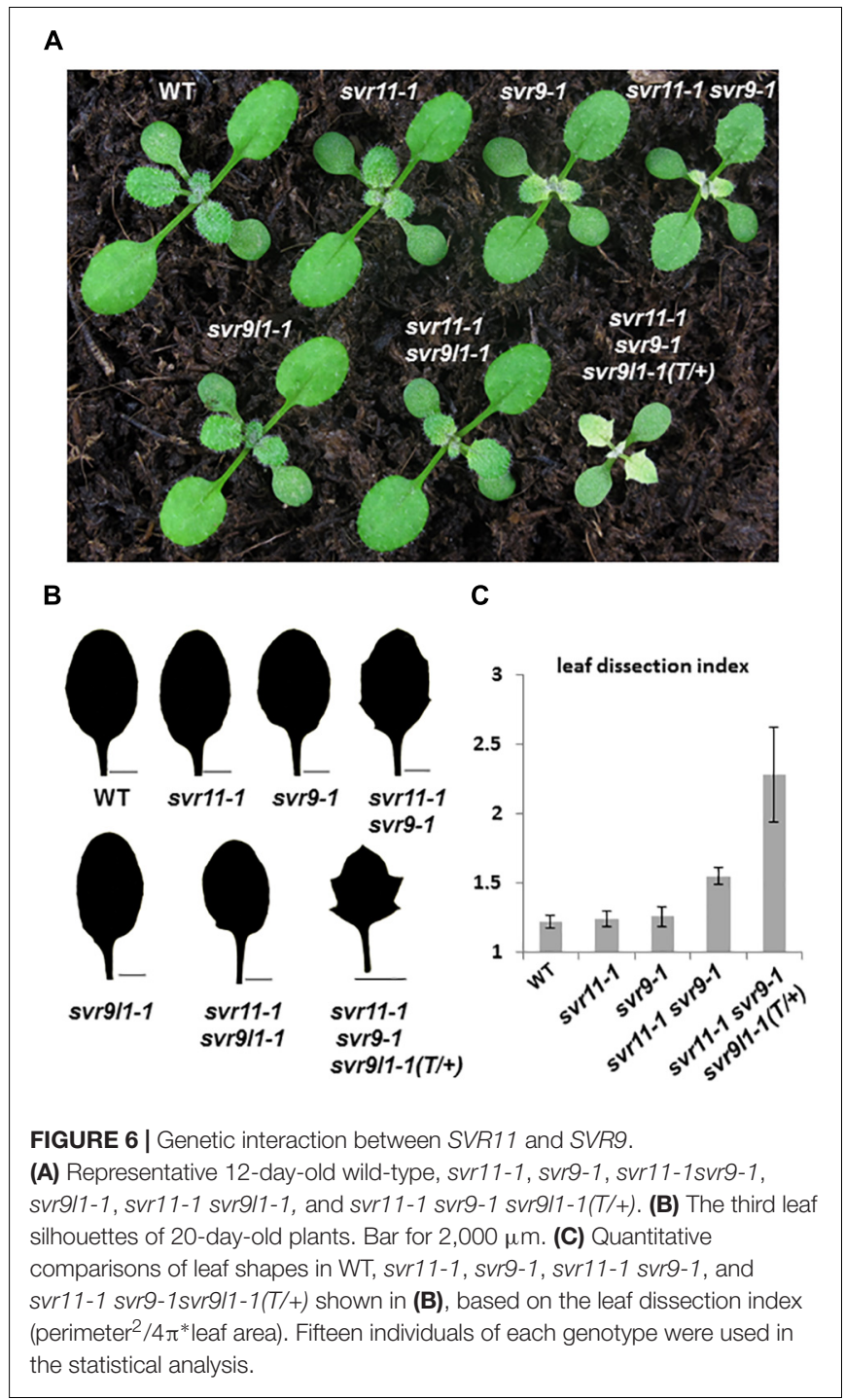

the functional relationships between SVR11 and SVR9, as well as SVR9L1, a functionally redundant homolog of SVR9 (Figure 6) (Zheng et al., 2016). At the single mutant level, svr9-1 showed a stronger degree of virescence than that of svr11-1, and svr9l1-1 showed a WT-like phenotype, as reported (Figure 6A) (Zheng et al., 2016). Consistent with both EF-Tu and IF3's involvement in translation, svr11-1 svr91 mutants were more virescent (Figure 6). The virescent level of svr11-1, svr9-1 and svr11-1 svr9-1 double mutants were quantified by measurement of $F_{\mathrm{V}} / F_{\mathrm{M}}$ (Supplementary Figure S3). Interestingly, svr11-1 svr9-1, but not svr11-1 svr9l1-1, showed a prominent leaf margin serration phenotype (Figure 6B). Furthermore, we obtained mutants that are homozygous for svr11-1 and svr9-1 while heterozygous for svr9l1-1 (svr11-1 svr9-1 svr9l1-1 T/+). These mutants not only showed strong virescence phenotype, the leaf serration was also the most conspicuous (Figures 6B,C). The leaf serrations were further quantified by the leaf dissection index (perimeter ${ }^{2} / 4 \pi^{*}$ leaf area) (Bilsborough et al., 2011) (Figure 6C).

We have shown that leaf serration phenotype may be associated with leaf vasculature development (Zheng et al., 2016). We then tested leaf vascular development and examined the cotyledon venation patterns in svr11-1 and svr11-1 svr9-1 double mutant. The numbers of closed areoles in mature cotyledons are indicators of leaf vascular development (Sieburth, 1999), and cotyledons from 10-day-old seedlings were observed under a dissecting microscope. Wild type cotyledons with two, three, and four areoles were predominant (Zheng et al., 2016, and also in the research, Table 2). In svr11-1, although the similar percentage of cotyledons show two, three or four areoles compared to wild type, cotyledons with only one areole were also identified in svr11-1 (Table 2). Noticeably, cotyledons with only one areole or no closed areoles were drastically increased in svr11-1 svr91 (Figure 7 and Table 2). Taken together, these data show that chloroplast translation EF-Tu and IF3 activities act synergistically to regulate leaf margin and cotyledon vascular development.

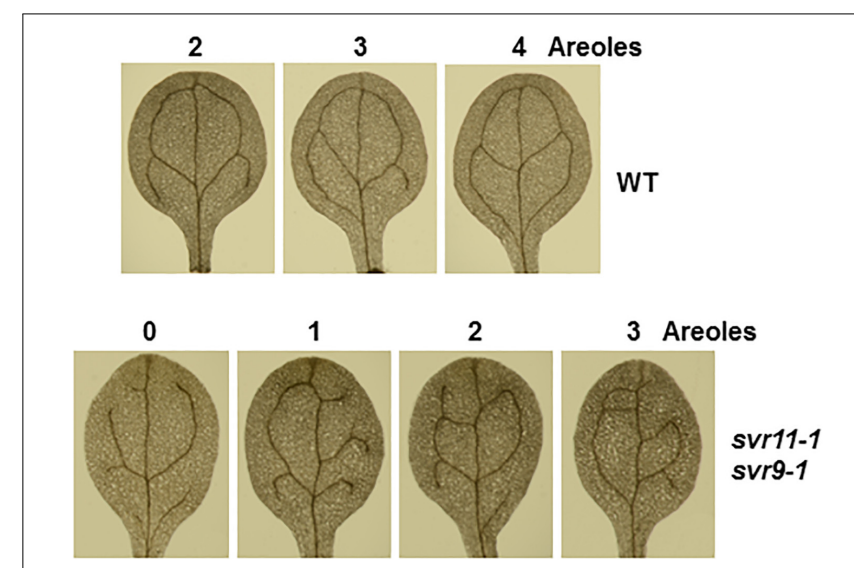

FIGURE 7 | Representative 10-day-old cotyledon vein patterns with different numbers of areoles from wild-type and svr11-1 svr9-1.

TABLE 2 | Quantification of cotyledon vein patterns in wild type, svr11-1 and svr11-1 svr9-1.

\begin{tabular}{|c|c|c|c|c|c|c|c|}
\hline Genotype & Total & Zero areole & One areole & Two areoles & Three areoles & Four areoles & Five areoles \\
\hline WT & 353 & N.A. & N.A. & 171 (48.4\%) & 134 (38.0\%) & 48 (13.6\%) & N.A. \\
\hline svr11-1 & 432 & N.A. & $21(4.8 \%)$ & 194 (44.9\%) & 167 (38.7\%) & $50(11.6 \%)$ & N.A. \\
\hline svr11-1 svr9-1 & 345 & 56 (16.2\%) & 147 (42.6\%) & 105 (30.4\%) & 32 (9.3\%) & $5(1.4 \%)$ & N.A. \\
\hline
\end{tabular}

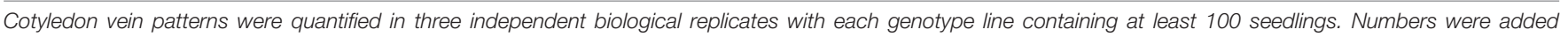
together from three independent biological replicates. Percentages of different types of areoles are indicated in the parentheses. N.A., not applicable. 


\section{DISCUSSION}

Chloroplasts are semi-autonomous organelles that derived from ancient cyanobacterium-like organisms through the process of endosymbiosis (Nowack and Weber, 2018). One of the key evidence supporting the endosymbiosis theory is the discovery of prokaryotic gene expression systems in the chloroplast. In this study, we identified svr11-1 as a genetic suppressor of the Arabidopsis var2 mutant and confirmed that SVR11 codes for a chloroplast-localized prokaryotic translation elongation factor EF-Tu. As a prokaryotic elongation factor, GTP-bound EF-Tu facilitates the transport of aminoacyl-tRNA to the A-site of the 70S ribosome during translation elongation (Krab and Parmeggiani, 2002). Consistent with their critical roles in translation, null alleles or higher-order mutants of many translation factors are lethal, suggesting essential roles of these factors for plant survival (Miura et al., 2007; Bryant et al., 2011; Zheng et al., 2016). Interestingly, svr11-1 mutant is phenotypically reminiscent of svr9-1, defective in a chloroplast translation initiation factor IF3 (Zheng et al., 2016). Both mutants show a distinct virescent phenotype with more pronounced reductions of chlorophyll accumulation and photosynthetic capacities at young, dividing tissues, suggesting their activities are required at early process (Lopez-Juez and Pyke, 2005). This is in agreement with the higher expression of these genes in young tissues and higher needs for their activities in those tissues. It is conceivable that a higher demand for translation capacities at younger stage of development is necessary to establish robust phototropic growth. Findings from our group and others have shown that mutations in chloroplast $70 \mathrm{~S}$ ribosome proteins mostly lead to rather uniform pale green or pale yellow leaf colorations (Bryant et al., 2011; Romani et al., 2012; Tiller et al., 2012; Liu et al., 2013). These findings suggest a possible regulatory way for translation factors and ribosomal proteins during chloroplast and leaf development at early stages.

Thylakoid-localized FtsHs are ATP-dependent zinc metalloproteases participating in the degradation of damaged photosynthetic subunits, especially damaged PSII reaction center D1 subunits during photoinhibition (Nishimura et al., 2016). Arabidopsis mutants var1 and var2, defective in thylakoidlocalized FtsH proteins VAR1/AtFtsH5 and VAR2/AtFtsH2, respectively, show unique leaf variegation phenotypes, suggesting that these FtsHs may play additional roles in chloroplast development besides D1 degradation (Chen et al., 2000; Takechi et al., 2000). Molecular genetics analyses of var2 suppressor mutants have also established that the chloroplast development defect, i.e., the leaf variegation phenotype, of var2 is dependent on functional chloroplast gene expression, especially chloroplast translation, providing further support for additional roles of VAR2/AtFtsH2 in chloroplast development (Miura et al., 2007; Yu et al., 2008; Liu et al., 2010, 2013; Putarjunan et al., 2013). Recently, we reported two var2 suppressor loci, SVR10, coding for a member of circularly permuted GTPase family involved in the processing of plastid ribosomal RNAs, and SVR9, a chloroplast translation initiation factor IF3 involved in chloroplast translation (Kim et al., 2012;
Qi et al., 2016; Zheng et al., 2016). In this report, building on the var2 suppressor screening work, we found that a mutation in SVR11, encoding a chloroplast translation elongation factor EF-Tu, can suppress var2 leaf variegated phenotype. The identification of SVR11 as a var2 suppression locus on one hand strengthens the functional relationship between VAR2/AtFtsH2 and chloroplast translation, on the other hand, it also provides further indication that VAR2/AtFtsH2 is related to the chloroplast translation process in general, rather than a specific functional link with individual components. Interestingly, through genetic enhancer analysis, we have recently for the first time established an intriguing link between VAR2/AtFtsH2 and cytosolic translation (Wang et al., 2018). In contrary to chloroplast translation, the reduction of cytosolic translation dramatically enhances var2 leaf variegation, suggesting chloroplast development in var2 is intimately regulated by cytosolic translation. Based on the suppression by reduced chloroplast translation and the enhancement by reduced cytosolic translation, a model was proposed in which FtsH may serve as an important factor in mediating the balance of cytosolic and chloroplast translation (Wang et al., 2018). Although the molecular mechanism underlying the maintenance of this balance remains unclear in higher plants, nuclear and mitochondrial translation balance has been shown to be vital of protein homeostasis in other model organisms (Topf et al., 2016).

The coordination between the nuclear and the chloroplast genome and gene expression requires fine regulation of bidirectional communications from nucleus to chloroplasts (anterograde) and also from chloroplasts to the nucleus (retrograde) (Jarvis and López-Juez, 2013). Given the importance and complexity, it came as no surprise that multiple regulatory pathways have been uncovered to ensure the coupling of the genomes. Canonical work have used the expressions of nuclear encoded photosynthetic genes, for example $L h c B$ or $R b c S$, as marker genes to probe the retrograde regulation of these genes when chloroplast functional states were disturbed, for instance, when treated with photo-bleaching herbicide norflurazon or chloroplast translation inhibitor lincomycin (Nott et al., 2006; Chi et al., 2013; Kleine and Leister, 2016). It has also been long recognized that the retrograde regulation of nuclear gene expression also involves the modulation of leaf development by the functional state of chloroplasts (Pogson et al., 2015). For example, abnormal leaf mesophyll developments were observed in the white leaf sectors of Arabidopsis immutans mutant, or in white tissues after norflurazon treatment (Aluru et al., 2009). Despite the accumulating evidence, our understanding of how states of chloroplasts affect leaf development remains limited. We previously reported that the chloroplast translation initiation factor IF3/SVR9 regulates chloroplast development, as well as leaf development, including leaf margin and cotyledon vasculature development (Zheng et al., 2016). In addition, we reported that mutations in SVR9 affect auxin homeostasis, and leaf margin development in a CUC2-dependent way (Nikovics et al., 2006; Zheng et al., 2016). In this work, we found that SVR11 also regulate leaf margin and cotyledon venation. 
Moreover, SVR11 and SVR9 work synergistically to regulate leaf margin development and cotyledon venation patterns. These findings reinforce the notion that chloroplast translation defects can trigger a signaling pathway to regulate leaf development (Zheng et al., 2016). This pathway seems to be activated only by certain types of translation defects caused mainly by the lack of translation factors, as not all chloroplast translation mutant display related phenotypes. Recently, it was shown that VAR2/AtFtsH2 may mediate a singlet oxygen signaling pathway from chloroplasts to the nucleus (Wang et al., 2016). Future research is warranted to address the relationship between these pathways and the components of this signaling pathway from the chloroplast to the nucleus.

\section{AUTHOR CONTRIBUTIONS}

YQ and FY conceived and coordinated the study and wrote the manuscript. SL, LZ, JJ, JG, and MZ designed, performed, and analyzed the experiments shown in Figures 1-7. JZ, JS, LA, and XL provided technical assistance and contributed to the preparation of the figures. All authors reviewed the results and approved the final version of the manuscript.

\section{REFERENCES}

Adam, Z., Frottin, F., Espagne, C., Meinnel, T., and Giglione, C. (2011). Interplay between $\mathrm{N}$-terminal methionine excision and $\mathrm{FtsH}$ protease is essential for normal chloroplast development and function in Arabidopsis. Plant Cell 23, 3745-3760. doi: 10.1105/tpc.111.087239

Aluru, M. R., Zola, J., Foudree, A., and Rodermel, S. R. (2009). Chloroplast photooxidation-induced transcriptome reprogramming in Arabidopsis immutans white leaf sectors. Plant Physiol. 150, 904-923. doi: 10.1104/pp.109.135780

Bilsborough, G. D., Runions, A., Barkoulas, M., Jenkins, H. W., Hasson, A., Galinha, C., et al. (2011). Model for the regulation of Arabidopsis thaliana leaf margin development. Proc. Natl. Acad. Sci. U.S.A. 108, 3424-3429. doi: 10.1073/pnas. 1015162108

Bryant, N., Lloyd, J., Sweeney, C., Myouga, F., and Meinke, D. (2011). Identification of nuclear genes encoding chloroplast-localized proteins required for embryo development in Arabidopsis. Plant Physiol. 155, 1678-1689. doi: 10.1104/pp. 110.168120

Chen, M., Choi, Y., Voytas, D. F., and Rodermel, S. (2000). Mutations in the Arabidopsis VAR2 locus cause leaf variegation due to the loss of a chloroplast FtsH protease. Plant J. 22, 303-313. doi: 10.1046/j.1365-313x.2000.00738.x

Chi, W., Sun, X., and Zhang, L. (2013). Intracellular signaling from plastid to nucleus. Annu. Rev. Plant Biol. 64, 559-582. doi: 10.1146/annurev-arplant050312-120147

Clough, S. J., and Bent, A. F. (1998). Floral dip: a simplified method for Agrobacterium-mediated transformation of Arabidopsis thaliana. Plant J. 16, 735-743. doi: 10.1046/j.1365-313x.1998.00343.x

Emanuelsson, O., Nielsen, H., and von Heijne, G. (1999). ChloroP, a neural network-based method for predicting chloroplast transit peptides and their cleavage sites. Protein Sci. 8, 978-984. doi: 10.1110/ps.8.5.978

Ito, J., Heazlewood, J. L., and Millar, A. H. (2006). Analysis of the soluble ATPbinding proteome of plant mitochondria identifies new proteins and nucleotide triphosphate interactions within the matrix. J. Proteome Res. 5, 3459-3469. doi: $10.1021 /$ pr060403j

Janska, H., Kwasniak, M., and Szczepanowska, J. (2013). Protein quality control in organelles - AAA/FtsH story. Biochim. Biophys. Acta 1833, 381-387. doi: 10.1016/j.bbamcr.2012.03.016

\section{FUNDING}

This work was supported by National Natural Science Foundation of China Grants 31741010 (to YQ), 31470290 (to LA), and 31770345 (to JS), and by Fundamental Research Funds for the Central Universities Grants 2452018152 (to YQ).

\section{SUPPLEMENTARY MATERIAL}

The Supplementary Material for this article can be found online at: https://www.frontiersin.org/articles/10.3389/fpls.2019.00295/ full\#supplementary-material

FIGURE S1 | Transient expression of p35S::SVR11-like-GFP fusion protein in leaf protoplasts of plants expressing the mitochondrion marker ScCOX4-mCherry. Representative images of a single protoplast are shown. Bar stands for $10 \mu \mathrm{m}$.

FIGURE S2 | Representative seedlings treated with a moderate level heat stress. Eight-day-old seedlings grown at $22^{\circ} \mathrm{C}$ were treated at $38^{\circ} \mathrm{C}$ for $90 \mathrm{~min}$, and then returned into $22^{\circ} \mathrm{C}$ for recovery. After 6 days recovery, seedlings were photographed.

FIGURE S3 | Representative 2-week-old seedlings and the corresponding $F_{\mathrm{V}} / F_{\mathrm{M}}$ images of wild type, svr11-1, svr9-1, and the double mutant svr11-1 svr9-1. The average value of $F_{\mathrm{O}}, F_{\mathrm{M}}, F_{\mathrm{V}} / F_{\mathrm{M}}$ were calculated from at least 10 individuals from each genotype.

Jarvis, P., and López-Juez, E. (2013). Biogenesis and homeostasis of chloroplasts and other plastids. Nat. Rev. Mol. Cell Biol. 14, 787-802. doi: 10.1038/nrm3702

Jefferson, R. A., Kavanagh, T. A., and Bevan, M. W. (1987). GUS fusions: betaglucuronidase as a sensitive and versatile gene fusion marker in higher plants. EMBO J. 6, 3901-3907. doi: 10.1002/j.1460-2075.1987.tb02730.x

Kato, Y., Miura, E., Ido, K., Ifuku, K., and Sakamoto, W. (2009). The variegated mutants lacking chloroplastic FtsHs are defective in D1 degradation and accumulate reactive oxygen species. Plant Physiol. 151, 1790-1801. doi: 10.1104/ pp.109.146589

Kim, B. H., Malec, P., Waloszek, A., and von Arnim, A. G. (2012). Arabidopsis BPG2: a phytochrome-regulated gene whose protein product binds to plastid ribosomal RNAs. Planta 236, 677-690. doi: 10.1007/s00425-012-1638-6

Kleine, T., and Leister, D. (2016). Retrograde signaling: organelles go networking. Biochim. Biophys. Acta 1857, 1313-1325. doi: 10.1016/j.bbabio.2016.03.017

Krab, I. M., and Parmeggiani, A. (1998). EF-Tu, a GTPase odyssey. Biochim. Biophys. Acta 1443, 1-22. doi: 10.1016/S0167-4781(98)00169-9

Krab, I. M., and Parmeggiani, A. (2002). Mechanisms of EF-Tu, a pioneer GTPase. Prog. Nucleic Acid Res. Mol. Biol. 71, 513-551. doi: 10.1016/S0079-6603(02) 71050-7

Kumar, S., Stecher, G., Li, M., Knyaz, C., and Tamura, K. (2018). MEGA X: molecular evolutionary genetics analysis across computing platforms. Mol. Biol. Evol. 35, 1547-1549. doi: 10.1093/molbev/msy096

Laursen, B. S., Sørensen, H. P., Mortensen, K. K., and Sperling-Petersen, H. U. (2005). Initiation of protein synthesis in bacteria. Microbiol. Mol. Biol. Rev. 69, 101-123. doi: 10.1128/MMBR.69.1.101-123.2005

Li, X., Cai, C., Wang, Z., Fan, B., Zhu, C., and Chen, Z. (2018). Plastid translation elongation factor tu is prone to heat-induced aggregation despite its critical role in plant heat tolerance. Plant Physiol. 176, 3027-3045. doi: 10.1104/pp.17.01672

Lindahl, M., Spetea, C., Hundal, T., Oppenheim, A. B., Adam, Z., and Andersson, B. (2000). The thylakoid FtsH protease plays a role in the light-induced turnover of the photosystem II D1 protein. Plant Cell 12, 419-431. doi: 10.1105/tpc.12.3.419

Liu, X., Rodermel, S. R., and Yu, F. (2010). A var2 leaf variegation suppressor locus, SUPPRESSOR OF VARIEGATION3, encodes a putative chloroplast translation elongation factor that is important for chloroplast development in the cold. BMC Plant Biol. 10:287. doi: 10.1186/1471-2229-10-287

Liu, X., Zheng, M., Wang, R., Wang, R., An, L., Rodermel, S. R., et al. (2013). Genetic interactions reveal that specific defects of chloroplast translation are 
associated with the suppression of var2-mediated leaf variegation. J. Integr. Plant Biol. 55, 979-993. doi: 10.1111/jipb.12078

Lopez-Juez, E., and Pyke, K. A. (2005). Plastids unleashed: their development and their integration in plant development. Int. J. Dev. Biol. 49, 557-577. doi: $10.1387 / \mathrm{ijdb} .051997 \mathrm{el}$

Malnoë, A., Wang, F., Girard-Bascou, J., Wollman, F. A., and de Vitry, C. (2014). Thylakoid FtsH protease contributes to photosystem II and cytochrome b6f remodeling in Chlamydomonas reinhardtii under stress conditions. Plant Cell 26, 373-390. doi: 10.1105/tpc.113.120113

Martin, W., Rujan, T., Richly, E., Hansen, A., Cornelsen, S., Lins, T., et al. (2002). Evolutionary analysis of Arabidopsis, cyanobacterial, and chloroplast genomes reveals plastid phylogeny and thousands of cyanobacterial genes in the nucleus. Proc. Natl. Acad. Sci. U.S.A. 99, 12246-12251. doi: 10.1073/pnas.182432999

Miura, E., Kato, Y., Matsushima, R., Albrecht, V., Laalami, S., and Sakamoto, W. (2007). The balance between protein synthesis and degradation in chloroplasts determines leaf variegation in Arabidopsis yellow variegated mutants. Plant Cell 19, 1313-1328. doi: 10.1105/tpc. 106.049270

Nelson, B. K., Cai, X., and Nebenführ, A. (2007). A multicolored set of in vivo organelle markers for co-localization studies in Arabidopsis and other plants. Plant J. 51, 1126-1136. doi: 10.1111/j.1365-313X.2007.03212.x

Nesbit, A. D., Whippo, C., Hangarter, R. P., and Kehoe, D. M. (2015). Translation initiation factor 3 families: what are their roles in regulating cyanobacterial and chloroplast gene expression? Photosynth. Res. 126, 147-159. doi: 10.1007/ s11120-015-0074-4

Nikolovski, N., Rubtsov, D., Segura, M. P., Miles, G. P., Stevens, T. J., Dunkley, T. P., et al. (2012). Putative glycosyltransferases and other plant Golgi apparatus proteins are revealed by LOPIT proteomics. Plant Physiol. 160, 1037-1051. doi: 10.1104/pp.112.204263

Nikovics, K., Blein, T., Peaucelle, A., Ishida, T., Morin, H., Aida, M., et al. (2006). The balance between the MIR164A and CUC2 genes controls leaf margin serration in Arabidopsis. Plant Cell 18, 2929-2945. doi: 10.1105/tpc.106.045617

Nishimura, K., Kato, Y., and Sakamoto, W. (2016). Chloroplast proteases: updates on proteolysis within and across suborganellar compartments. Plant Physiol. 171, 2280-2293. doi: 10.1104/pp.16.00330

Nott, A., Jung, H. S., Koussevitzky, S., and Chory, J. (2006). Plastid-to-nucleus retrograde signaling. Annu. Rev. Plant Biol. 57, 739-759. doi: 10.1146/annurev. arplant.57.032905.105310

Nowack, E. C. M., and Weber, A. P. M. (2018). Genomics-informed insights into endosymbiotic organelle evolution in photosynthetic eukaryotes. Annu. Rev. Plant Biol. 69, 51-84. doi: 10.1146/annurev-arplant-042817-040209

Park, S., and Rodermel, S. R. (2004). Mutations in ClpC2/Hsp100 suppress the requirement for FtsH in thylakoid membrane biogenesis. Proc. Natl. Acad. Sci. U.S.A. 101, 12765-12770. doi: 10.1073/pnas.0402764101

Pogson, B. J., Ganguly, D., and Albrecht-Borth, V. (2015). Insights into chloroplast biogenesis and development. Biochim. Biophys. Acta 1847, 1017-1024. doi: 10.1016/j.bbabio.2015.02.003

Putarjunan, A., Liu, X., Nolan, T., Yu, F., and Rodermel, S. (2013). Understanding chloroplast biogenesis using second-site suppressors of immutans and var2. Photosynth. Res. 116, 437-453. doi: 10.1007/s11120-013-9855-9

Qi, Y., Zhao, J., An, R., Zhang, J., Liang, S., Shao, J., et al. (2016). Mutations in circularly permuted GTPase family genes AtNOA1/RIF1/SVR10 and BPG2 suppress var2-mediated leaf variegation in Arabidopsis thaliana. Photosynth. Res. 127, 355-367. doi: 10.1007/s11120-015-0195-9

Queitsch, C., Hong, S. W., Vierling, E., and Lindquist, S. (2000). Heat shock protein 101 plays a crucial role in thermotolerance in Arabidopsis. Plant Cell 12, 479-492. doi: 10.1105/tpc.12.4.479

Ristic, Z., Wilson, K., Nelsen, C., Momcilovic, I., Kobayashi, S., Meeley, R., et al. (2004). A maize mutant with decreased capacity to accumulate chloroplast protein synthesis elongation factor (EF-Tu) displays reduced tolerance to heat stress. Plant Sci. 167, 1367-1374. doi: 10.1016/j.plantsci.2004.07.016

Romani, I., Tadini, L., Rossi, F., Masiero, S., Pribil, M., Jahns, P., et al. (2012). Versatile roles of Arabidopsis plastid ribosomal proteins in plant growth and development. Plant J. 72, 922-934. doi: 10.1111/tpj.12000

Rose, A. B., Elfersi, T., Parra, G., and Korf, I. (2008). Promoter-proximal introns in Arabidopsis thaliana are enriched in dispersed signals that elevate gene expression. Plant Cell 20, 543-551. doi: 10.1105/tpc.107.057190

Sakamoto, W., Tamura, T., Hanba-Tomita, Y., Murata, M., and Sodmergen. (2002). The VAR1 locus of Arabidopsis encodes a chloroplastic FtsH and is responsible for leaf variegation in the mutant alleles. Genes Cells 7, 769-780. doi: 10.1046/j. 1365-2443.2002.00558.x

Shen, Y., Li, C., McCarty, D. R., Meeley, R., and Tan, B. C. (2013). Embryo defective12 encodes the plastid initiation factor 3 and is essential for embryogenesis in maize. Plant J. 74, 792-804. doi: 10.1111/tpj.12161

Sieburth, L. E. (1999). Auxin is required for leaf vein pattern in Arabidopsis. Plant Physiol. 121, 1179-1190. doi: 10.1104/pp.121.4.1179

Takechi, K., Sodmergen, Murata, M., Motoyoshi, F., and Sakamoto, W. (2000). The YELLOW VARIEGATED (VAR2) locus encodes a homologue of FtsH, an ATP-dependent protease in Arabidopsis. Plant Cell Physiol. 41, 1334-1346. doi: $10.1093 /$ pcp/pcd067

Tiller, N., and Bock, R. (2014). The translational apparatus of plastids and its role in plant development. Mol Plant. 7, 1105-1120. doi: 10.1093/mp/ssu022

Tiller, N., Weingartner, M., Thiele, W., Maximova, E., Schöttler, M. A., and Bock, R. (2012). The plastid-specific ribosomal proteins of Arabidopsis thaliana can be divided into non-essential proteins and genuine ribosomal proteins. Plant J. 69, 302-316. doi: 10.1111/j.1365-313X.2011.04791.x

Timmis, J. N., Ayliffe, M. A., Huang, C. Y., and Martin, W. (2004). Endosymbiotic gene transfer: organelle genomes forge eukaryotic chromosomes. Nat. Rev. Genet. 5, 123-135. doi: 10.1038/nrg1271

Topf, U., Wrobel, L., and Chacinska, A. (2016). Chatty mitochondria: keeping balance in cellular protein homeostasis. Trends Cell Biol. 26, 577-586. doi: 10.1016/j.tcb.2016.03.002

Wang, L., Kim, C., Xu, X., Piskurewicz, U., Dogra, V., Singh, S., et al. (2016). Singlet oxygen- and EXECUTER1-mediated signaling is initiated in grana margins and depends on the protease FtsH2. Proc. Natl. Acad. Sci. U.S.A. 113, E3792-E3800. doi: 10.1073/pnas.1603562113

Wang, R., Zhao, J., Jia, M., Xu, N., Liang, S., Shao, J., et al. (2018). Balance between cytosolic and chloroplast translation affects leaf variegation. Plant Physiol. 176, 804-818. doi: 10.1104/pp.17.00673

Xiang, C., Han, P., Lutziger, I., Wang, K., and Oliver, D. J. (1999). A mini binary vector series for plant transformation. Plant Mol. Biol. 40, 711-717. doi: 10. 1023/a:1006201910593

Yamaguchi, K., and Subramanian, A. R. (2000). The plastid ribosomal proteins. Identification of all the proteins in the $50 \mathrm{~S}$ subunit of an organelle ribosome (chloroplast). J. Biol. Chem. 275, 28466-28482. doi: 10.1074/jbc.M005012200

Yamaguchi, K., von Knoblauch, K., and Subramanian, A. R. (2000). The plastid ribosomal proteins. Identification of all the proteins in the $30 \mathrm{~S}$ subunit of an organelle ribosome (chloroplast). J. Biol. Chem. 275, 28455-28465. doi: 10.1074/ jbc.M004350200

Yoo, S. D., Cho, Y. H., and Sheen, J. (2007). Arabidopsis mesophyll protoplasts: a versatile cell system for transient gene expression analysis. Nat. Protoc. 2, 1565-1572. doi: 10.1038/nprot.2007.199

Yu, F., Liu, X., Alsheikh, M., Park, S., and Rodermel, S. (2008). Mutations in SUPPRESSOR OF VARIEGATION1, a factor required for normal chloroplast translation, suppress var2-mediated leaf variegation in Arabidopsis. Plant Cell 20, 1786-1804. doi: 10.1105/tpc. 107.054965

Yu, F., Park, S., and Rodermel, S. R. (2004). The Arabidopsis FtsH metalloprotease gene family: interchangeability of subunits in chloroplast oligomeric complexes. Plant J. 37, 864-876. doi: 10.1111/j.1365-313X.2003.02014.x

Zaltsman, A., Ori, N., and Adam, Z. (2005). Two types of FtsH protease subunits are required for chloroplast biogenesis and Photosystem II repair in Arabidopsis. Plant Cell 17, 2782-2790. doi: 10.1105/tpc.105.035071

Zheng, M., Liu, X., Liang, S., Fu, S., Qi, Y., Zhao, J., et al. (2016). Chloroplast translation initiation factors regulate leaf variegation and development. Plant Physiol. 172, 1117-1130. doi: 10.1104/pp.15.02040

Conflict of Interest Statement: The authors declare that the research was conducted in the absence of any commercial or financial relationships that could be construed as a potential conflict of interest.

Copyright $\odot 2019$ Liu, Zheng, Jia, Guo, Zheng, Zhao, Shao, Liu, An, Yu and Qi. This is an open-access article distributed under the terms of the Creative Commons Attribution License (CC BY). The use, distribution or reproduction in other forums is permitted, provided the original author(s) and the copyright owner(s) are credited and that the original publication in this journal is cited, in accordance with accepted academic practice. No use, distribution or reproduction is permitted which does not comply with these terms. 